\title{
Comparison of heterologous xylose transporters in recombinant Saccharomyces cerevisiae
}

\author{
David Runquist, Bärbel Hahn-Hägerdal, Peter Rådström
}

\begin{abstract}
Background: Baker's yeast (Saccharomyces cerevisiae) has been engineered for xylose utilization to enable production of fuel ethanol from lignocellulose raw material. One unresolved challenge is that $S$. cerevisiae lacks a dedicated transport system for pentose sugars, which means that xylose is transported by non-specific Hxt transporters with comparatively low transport rate and affinity for xylose.

Results: In this study, we compared three heterologous xylose transporters that have recently been shown to improve xylose uptake under different experimental conditions. The transporters Gxf1, Sut1 and At5g59250 from Candida intermedia, Pichia stipitis and Arabidopsis thaliana, respectively, were expressed in isogenic strains of $S$. cerevisiae and the transport kinetics and utilization of xylose was evaluated. Expression of the Gxf1 and Sut1 transporters led to significantly increased affinity and transport rates of xylose. In batch cultivation at $4 \mathrm{~g} / \mathrm{L}$ xylose concentration, improved transport kinetics led to a corresponding increase in xylose utilization, whereas no correlation could be demonstrated at xylose concentrations greater than $15 \mathrm{~g} / \mathrm{L}$. The relative contribution of native sugar transporters to the overall xylose transport capacity was also estimated during growth on glucose and xylose.

Conclusions: Kinetic characterization and aerobic batch cultivation of strains expressing the Gxf1, Sut1 and At5g59250 transporters showed a direct relationship between transport kinetics and xylose growth. The Gxf1 transporter had the highest transport capacity and the highest xylose growth rate, followed by the Sut1 transporter. The range in which transport controlled the growth rate was determined to between 0 and $15 \mathrm{~g} / \mathrm{L}$ xylose. The role of catabolite repression in regulation of native transporters was also confirmed by the observation that xylose transport by native $S$. cerevisiae transporters increased significantly during cultivation in xylose and at low glucose concentration.
\end{abstract}

\section{Background}

Baker's yeast (Saccharomyces cerevisiae) is used for industrial production of fuel ethanol from starch (corn) and sucrose (sugar cane). Lignocellulose biomass derived from forest and agricultural byproducts has been investigated as an alternative substrate, which does not compete with food and feed production [1,2]. Lignocellulose biomass is a complex substrate composed of hexose and pentose sugar polymers. Native $S$. cerevisiae is unable to utilize pentose sugars and considerable research has been devoted to enable utilization of xylose, which is the predominant pentose sugar in lignocellulose. Xylose utilization in S. cerevisiae has been accomplished by expression of the enzymes xylose reductase (XR) and

\footnotetext{
* Correspondence: Peter.Radstrom@tmb.lth.se

Applied Microbiology, Lund University, PO Box 124, SE-221 00 Lund, Sweden
}

xylitol dehydrogenase $(\mathrm{XDH})$ or by expression of xylose isomerase (XI) [3-5].

In recombinant xylose-utilizing $S$. cerevisiae, xylose is transported by non-specific hexose transporters with poor affinity for xylose [6,7]. The degree to which transport controls xylose utilization in S. cerevisiae is dependent on the flux in the downstream pathway and the concentration of xylose in the medium $[8,9]$. The first constructed xylose-utilizing $S$. cerevisiae strains were not constrained by transport because of their slow xylose catabolism [10], whereas current recombinant S. cerevisiae strains are transport-constrained, at least at low substrate concentrations [9]. There have been recent reports of improved transport and utilization of xylose in S. cerevisiae by independent expression of the heterologous xylose transporters Gxf1 [9], Sut1 [11] and 
At5g5920 [12] from Candida intermedia, Pichia stipitis and Arabidopsis thaliana, respectively. However, these investigations were performed using different strains and experimental setups, which limits a comparative evaluation of the transporters and their effect on xylose utilization.

In the current study, the transporters Gxf1, Sut1 and At5g5920 were expressed in isogenic strains and assessed under identical growth conditions. Xylose transport kinetics were determined and related to aerobic xylose growth at different substrate concentrations. In addition, the contribution of native $S$. cerevisiae transporters to the overall xylose transport was estimated during growth on glucose and xylose at different concentrations. Results showed a direct relationship between xylose transport kinetics and the xylose growth rate during transport-limited conditions.

\section{Methods}

\section{Strains and cultivation conditions}

Plasmids and S. cerevisiae strains used in this study are summarized in Table 1. Escherichia coli strain DH $5 \alpha$ was used for subcloning and was grown on lysogeny broth (LB) agar plates supplemented with $100 \mathrm{mg} / \mathrm{L}$ ampicillin. Defined mineral medium was used for $S$. cerevisiae cultivation (carbon source (glucose or xylose); mineral salts $\left(5 \mathrm{~g} / \mathrm{L}\left(\mathrm{NH}_{4}\right)_{2} \mathrm{SO}_{4}, 3 \mathrm{~g} / \mathrm{L} \mathrm{KH}_{2} \mathrm{PO}_{4}, 0.5 \mathrm{~g} / \mathrm{L}\right.$ $\left.\mathrm{MgSO}_{4} \cdot 7 \mathrm{H}_{2} \mathrm{O}\right)$; vitamins and trace elements [13] and buffering agent $(50 \mathrm{mM}$ potassium hydrogen phthalate, $\mathrm{pH}$ 5.5)) [14]. Yeast strains were streaked from $15 \% \mathrm{v} / \mathrm{v}$

\section{Table 1 Plasmids and strains used in the current study}

\begin{tabular}{|c|c|c|}
\hline $\begin{array}{l}\text { Strains and } \\
\text { plasmids }\end{array}$ & Relevant genotype & Reference \\
\hline \multicolumn{3}{|l|}{ Plasmids } \\
\hline Ylplac128 & LEU2 & [33] \\
\hline YlpOB9 & $\begin{array}{l}\text { URA3 TDH3p-XYL1(K27OR)-ADH1t, PGK1p- } \\
\text { XYL2-PGK1t }\end{array}$ & {$[19]$} \\
\hline pSUT1 & & Current study \\
\hline pAt5g5920 & & Current study \\
\hline YlpDR1 & Ylplac128 TDH3p-GXF1-CYC1t & {$[9]$} \\
\hline YlpDR4 & Ylplac128 TDH3p-SUT1-CYC1t & Current study \\
\hline YlpDR5 & Ylplac128 TDH3p-At5g5920-CYC1t & Current study \\
\hline \multicolumn{3}{|l|}{$\begin{array}{l}\text { S. cerevisiae } \\
\text { strains }\end{array}$} \\
\hline TMB 3043 & $\begin{array}{l}\text { CEN.PK 2-1C } \triangle \text { gre3, his3::PGK1p-XKS1- } \\
\text { PGK1t, TAL1::PGK1p-TAL1-PGK1t, TKL1:: } \\
\text { PGK1p-TKL1-PGK1t, RKI1::PGK1p-RKI1- } \\
\text { PGK1t, RPE1::PGK1p-RPE1-PGK1t, leu2, ura3 }\end{array}$ & {$[34]$} \\
\hline TMB 3662 & TMB 3043, ura3::YlpOB9 & {$[19]$} \\
\hline TMB 3415 & TMB 3662, leu2::Ylplac128 & Current study \\
\hline TMB 3416 & TMB 3662, leu2::YIpDR1 & Current study \\
\hline TMB 3418 & TMB 3662, leu2::YIpDR4 & Current study \\
\hline TMB 3419 & TMB 3662, leu2::YIpDR5 & Current study \\
\hline
\end{tabular}

glycerol stock and grown for 2 days on yeast nitrogen base (YNB) glucose plates at $30^{\circ} \mathrm{C}$. A single colony was pre-grown overnight to late exponential phase in mineral medium (20 g/L glucose) and used for subsequent inoculation. Cultivation of $S$. cerevisiae was performed at $30^{\circ} \mathrm{C}$.

\section{Strain construction}

Standard molecular biology techniques were used for all cloning procedures [15]. Plasmid purification was performed using a commercial kit (GeneJet Plasmid Miniprep Kit; Fermentas, Vilnius, Lithuania) and DNA was extracted from agarose gels (QIAquick Gel Extraction Kit; Qiagen, Hilden, Germany). Enzymes including T4 DNA ligase, calf intestine alkaline phosphatase, DreamTaq polymerase and various restriction enzymes were obtained from Fermentas. Transformation of E. coli and $S$. cerevisiae was performed using the calcium chloride [16] and the lithium acetate [17] methods, respectively.

The SUT1 gene from P. stipitis and the At5g59250gene from $A$. thaliana were codon-optimized for expression in $S$. cerevisiae by using the Codon Adaptation Index (CAI) and the Java Codon Adaptation Tool (JCat) [18]. The optimized SUT1 and At5g59250 genes were constructed synthetically (GenScript, Piscataway, NJ, USA), resulting in plasmids pSUT1 and pAt5g59250. The SUT1 and At5g59250 open reading frames (ORF) were isolated from plasmids pSUT1 and pAt5g59250 using the restriction enzymes SpeI and SalI followed by gel purification. The YIpDR4 integrative plasmid was constructed by inserting the SUT1 ORF between the TDH3 promoter and the CYC1 terminator of the previously constructed YIpDR1 vector (Table 1). YIpDR5 was similarly constructed using the excised At5g59250 ORF. The integrative plasmids YIpDR1, YIpDR4 and YIpDR5 were linearized using EcoRV, and transformed into S. cerevisiae strain TMB 3662 [19], yielding strains TMB 3416, TMB 3418 and TMB 3419 (Table 1). The control strain TMB 3415 was constructed by integration of plasmid YIplac128, not harboring any heterologous transporter gene, in TMB 3662 (Table 1).

\section{Transport kinetics}

Initial xylose uptake rates were determined using a previously described method [20] with minor modifications. Cells were inoculated into $1 \mathrm{~L}$ shake flasks in $100 \mathrm{~mL}$ medium (20 g/L glucose, $0.5 \mathrm{~g} / \mathrm{L}$ glucose or $60 \mathrm{~g} / \mathrm{L}$ xylose) at an optical density at $620 \mathrm{~nm}\left(\mathrm{OD}_{620}\right)$ of 0.35 , and were harvested by centrifugation (5000 g for 2 minutes) after approximately two replications $\left(\mathrm{OD}_{620}=1.5\right)$. The resulting cell pellet was washed in $10 \mathrm{~mL}$ potassium phosphate buffer $100 \mathrm{mM}$ (pH 6.8), centrifuged (5000 g for 2 minutes) and resuspended in $1 \mathrm{~mL}$ potassium phosphate buffer. Initial uptake rates were determined at $25^{\circ} \mathrm{C}$ using 
D- $\left[\mathrm{U}-{ }^{14} \mathrm{C}\right]$ labeled xylose (GE Healthcare, Buckinghamshire, UK; specific radioactivity $500-1000 \mathrm{cpm} / \mathrm{nmol}$ ). The reactions were initiated by mixing $20 \mu \mathrm{L}$ of buffered cell solution with $20 \mu \mathrm{L}$ of radioactive substrate, giving final substrate concentrations between 3 and $150 \mathrm{mM}$. The reaction was quenched after 20 seconds by addition of 3.5 $\mathrm{mL}$ ice-cold $100 \mathrm{mM}$ sorbitol solution. The resulting solution was immediately filtered through a $2.5 \mathrm{~cm}$ GF/C filter (Whatman, Maidstone, UK) and washed with $2 \times 50 \mathrm{~mL}$ ice-cold water. Washed filters were transferred to scintillation vials containing $5 \mathrm{~mL}$ scintillation cocktail (Optiphase Hisafe 2; Perkin Elmer, CT, USA) and the corresponding radioactivity was measured in a liquid scintillation counter (Tri-Carb; Perkin Elmer). The baseline radioactivity was obtained in parallel measurements by adding the radioactive substrate to the cell solution after the quenching solution had been added. All measurements were performed in biological duplicate for each strain and condition. Matlab software (Matlab R2007b; The MathWorks Inc., MA, USA) was used for non-linear regression analysis of kinetic data.

\section{Aerobic batch cultivation}

Yeast strains were inoculated in $25 \mathrm{~mL}$ xylose medium $(60 \mathrm{~g} / \mathrm{L}, 15 \mathrm{~g} / \mathrm{L}$ or $4 \mathrm{~g} / \mathrm{L})$ in $250 \mathrm{~mL}$ baffled shake flasks at a starting $\mathrm{OD}_{620}$ of 0.2 . Cells were grown at $30^{\circ} \mathrm{C}$ in an orbital shaker at $200 \mathrm{rpm}$. The growth rate was followed by measuring the optical density (OD) at 620 $\mathrm{nm}$. All measurements were performed in biological duplicate for each investigated strain and each condition.

\section{Results}

\section{Xylose transport kinetics}

Xylose transport kinetics were determined in the xyloseutilizing S. cerevisiae strain TMB 3662 [19] expressing the heterologous transporters Gxf1, Sut1 and At5g59250 from C. intermedia, P. stipitis and A. thaliana, respectively. Cells were grown in $20 \mathrm{~g} / \mathrm{L}$ glucose for two cell divisions, after which xylose transport was assayed as initial rate of $\mathrm{D}-\left[\mathrm{U}_{-}-{ }^{14} \mathrm{C}\right]$ xylose uptake. The measured xylose transport rate was plotted against substrate concentration and fitted to a single-component MichaelisMenten equation by non-linear regression (Figure 1, Table 2). Because the measured transport rate included both heterologous and native $S$. cerevisiae transporters, the determined kinetic constants refer to the overall cellular transport capacity. The ratio of the maximum transport rate, $\mathrm{V}_{\max }$ and the substrate affinity, $\mathrm{K}_{\mathrm{m}}$, has, despite some shortfalls been widely used as an overall measure of enzymatic performance [21,22], and was used here for quantitative comparison of the different transporters (Table 2). Expression of the C. intermedia Gxf1 transporter and the P. stipitis Sut1 transporter was

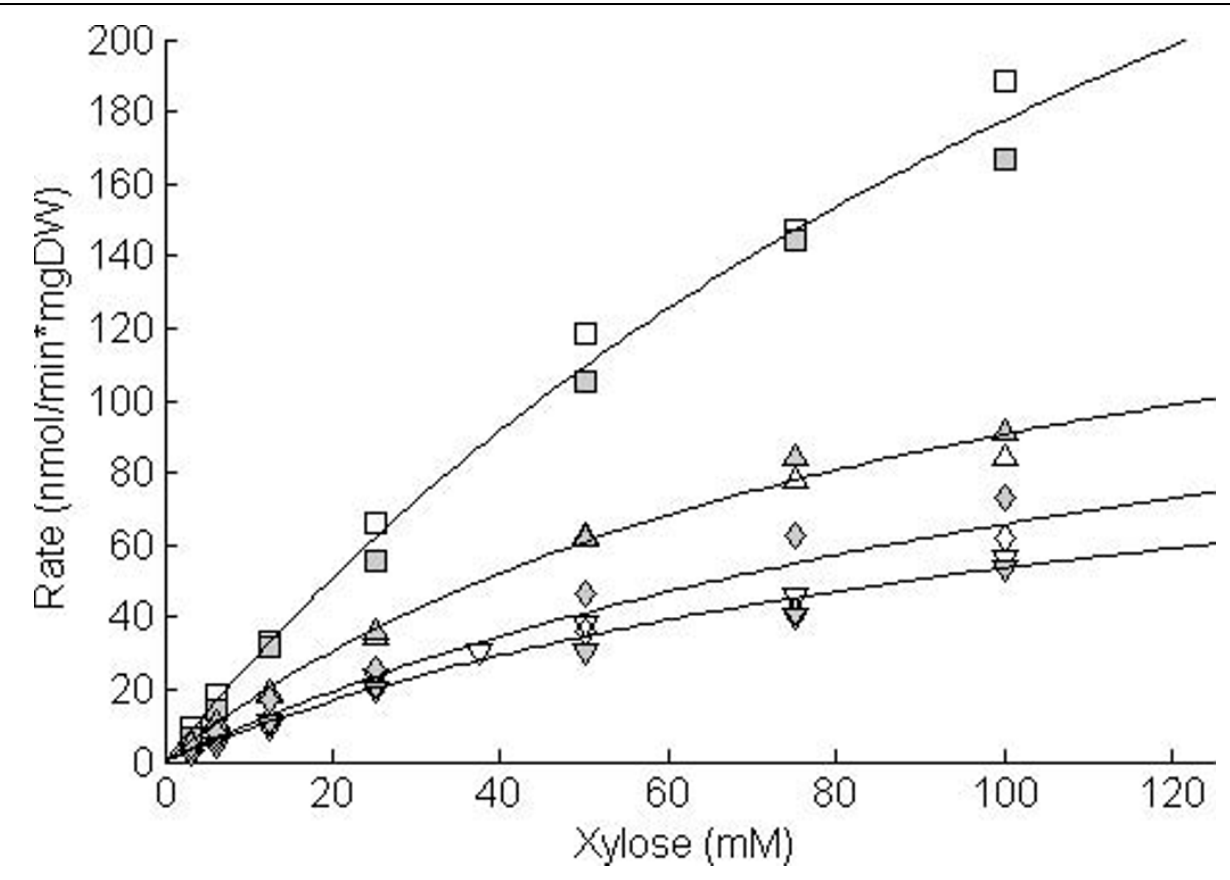

Figure 1 Xylose uptake rates of S. cerevisiae strains expressing the Gxf1, Sut1 and At5g59250 transporters determined using D-[U $\left.{ }^{14} \mathrm{C}\right]$ labeled sugar. Cells were grown on $20 \mathrm{~g} / \mathrm{L}$ glucose. Lines represent the calculated fit according to Michaelis-Menten kinetics. Gxf1, square; Sut1, upwards pointing triangle; At5g59250, diamond; control, downwards pointing triangle. Biological replicates are designated by empty and filled version of the same symbol. 
Table 2 Apparent kinetic constants for xylose transport in S. cerevisiae strains grown on 20 g/L glucose

\begin{tabular}{lccc}
\hline & $\mathbf{K}_{\mathbf{m}}, \mathbf{m M}$ & $\mathbf{V}_{\mathbf{m a x}}, \mathbf{n m o l} / \mathbf{m i n} \times \mathbf{m g D W}$ & $\mathbf{V}_{\mathbf{m a x}} / \mathbf{K}_{\mathbf{m}}, \mathbf{m i n}^{\mathbf{- 1}} \times \mathbf{m g D W}^{\mathbf{- 1}}$ \\
\hline TMB 3415 (control) & $121 \pm 44$ & $119 \pm 17$ & $10 \times 10^{-7}$ \\
TMB 3416 (Gxf1) & $166 \pm 6$ & $471 \pm 10$ & $28 \times 10^{-7}$ \\
TMB 3418 (Sut1) & $96 \pm 11$ & $178 \pm 16$ & $19 \times 10^{-7}$ \\
TMB 3419 (At5g59250) & $148 \pm 39$ & $163 \pm 7$ & $11 \times 10^{7}$ \\
\hline
\end{tabular}

thus seen to increase the transport performance compared with the reference strain expressing only native transporters (Figure 1, Table 2). In particular, the Gxf1 transporter increased the overall xylose transport performance by three times with respect to the control strain, which is comparable with results from previous studies $[9,23]$. A slight improvement was observed for the strain expressing the At5g59250 transporter, but it was not statistically significant (Figure 1, Table 2).

\section{Aerobic growth rates}

It has previously been shown that the effect of increased transport performance on xylose utilization is a function of strain background and substrate concentration $[8,9,24]$. In the current study, isogenic strains expressing the Gxf1, Sut1 and At5g59250 transporters were used to relate the measured transport kinetics to growth performance. The aerobic xylose growth rate of strains expressing the heterologous transporters was thus investigated at different substrate concentrations.

Strains expressing the Gxf1, Sut1 and At5g59250 transporters were first grown at high xylose concentration $(60 \mathrm{~g} / \mathrm{L})$. At this concentration, the strains expressing heterologous transporters and the control strain all displayed the same specific growth rate $(\mu), 0.16 \mathrm{~h}^{-1}$. Similarly, at intermediate xylose concentration $(15 \mathrm{~g} / \mathrm{L})$, all strains still displayed the same growth rate, but it was reduced to $0.04 \mathrm{~h}^{-1}$. At low xylose concentration $(4 \mathrm{~g} / \mathrm{L})$, the specific growth rate was further reduced to 0.002 to $0.008 \mathrm{~h}^{-1}$. However, at this concentration, strains expressing the heterologous transporters grew significantly faster than the control strain (Figure 2). The strain expressing the Gxf1 transporter displayed the highest growth rate, while the strain expressing the Sut1 transporter showed a small but reproducible increase in growth rate. The growth rate of the strain expressing

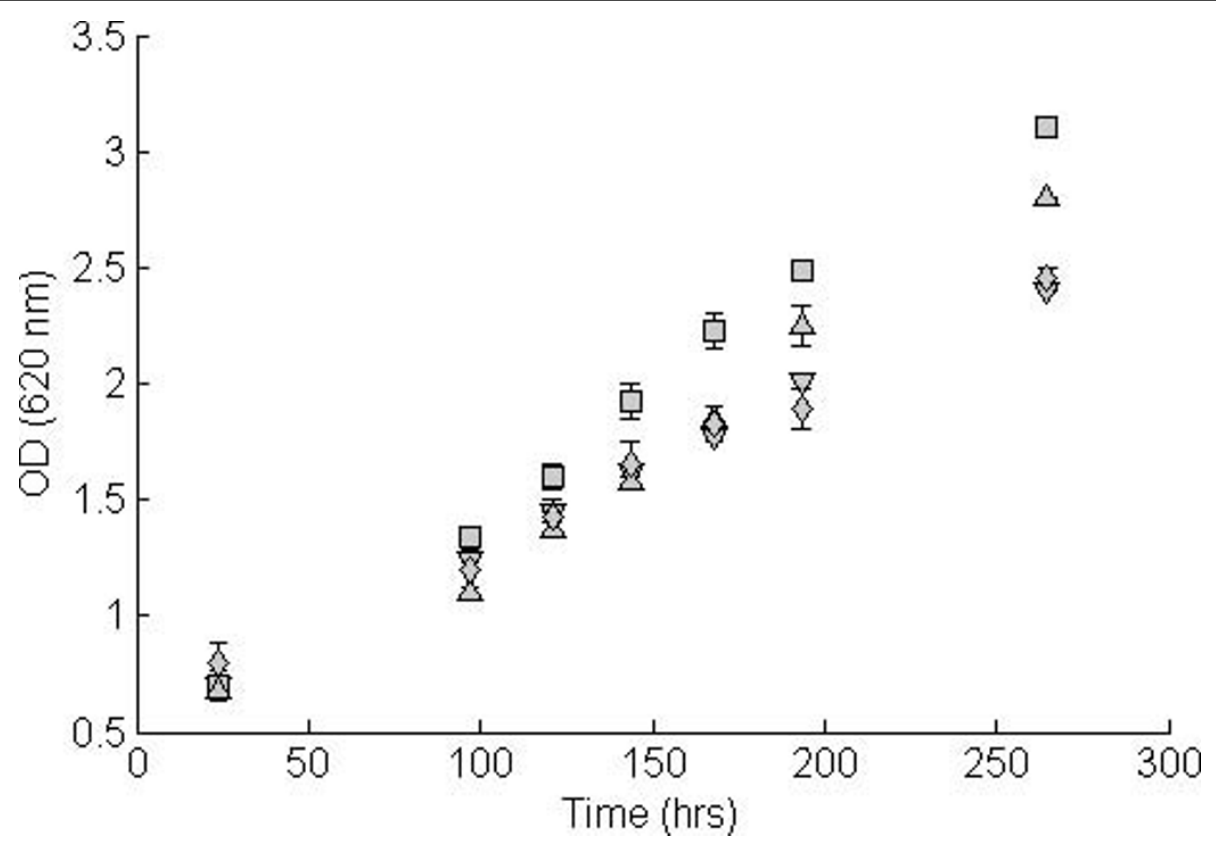

Figure 2 Aerobic batch cultivation xylose $4 \mathrm{~g} / \mathrm{L}$ of recombinant S. cerevisiae strains expressing the Gxf1, Sut1 and At5g59250 transporters. Gxf1, square; Sut1, upwards pointing triangle; At5959250, diamond; Control, downwards pointing triangle. Deviations between biological replicates are indicated by error bars. 
the At5g59250 transporter was the same as that of the control strain (Figure 2).

\section{Xylose transport by native $S$. cerevisiae transporters}

Kinetic characterization of $S$. cerevisiae strains expressing the Gxf1 and Sut1 transporters showed increased xylose transport performance compared with the control strain expressing only native transporters (Figure 1, Table 2). The performance of native S. cerevisiae transporters, as opposed to constitutively expressed heterologous transporters, depends on the type of transporters expressed in the cell, which in turn depends on the carbon source and its concentration $[7,25]$. The reported overall xylose transport affinity of native $S$. cerevisiae thus varies between 80 and $200 \mathrm{mM}$, depending on the cultivation conditions in the particular experiment $[6,7,10]$. In the current study, the effect of the cultivation condition was assayed by measuring the xylose transport kinetics of native $S$. cerevisiae transporters during cultivation on different concentrations of glucose and xylose.

TMB 3415, expressing only native transporters, was grown on $0.5 \mathrm{~g} / \mathrm{L}$ and $20 \mathrm{~g} / \mathrm{L}$ glucose and on $60 \mathrm{~g} / \mathrm{L}$ xylose, and was subsequently assayed for xylose transport (Figure 3). The xylose transport performance $\left(\mathrm{V}_{\max } / \mathrm{K}_{\mathrm{m}}\right)$ increased when cells were grown at $0.5 \mathrm{~g} / \mathrm{L}$ glucose $\left(28 \times 10-^{7} \mathrm{~min}^{-1} \times \mathrm{mgDW}^{1}{ }^{1}\right)$ and $60 \mathrm{~g} / \mathrm{L}$ xylose $\left(13 \times 10-^{7} \mathrm{~min}^{-1} \times \mathrm{mgDW}^{1}{ }^{1}\right)$ compared with $20 \mathrm{~g} / \mathrm{L}$ glucose $\left(10 \times 10-^{7} \min ^{-1} \times \mathrm{mgDW}^{-1}\right)$. Cultivation at the low glucose concentration $(0.5 \mathrm{~g} / \mathrm{L})$ yielded the highest xylose transport performance, which was comparable with cells expressing the Gxf1 transporter during cultivation on $20 \mathrm{~g} / \mathrm{L}$ glucose (Figure 1, Table 2). The results confirm those of previous studies, which showed increased expression of high affinity transporters and increased xylose transport during non-repressive conditions; that is, low glucose concentration $[7,25]$.

\section{Discussion}

Compared with glucose, the transport affinity for xylose in $S$. cerevisiae is very low $[6,7]$. It has thus been hypothesized that transport may control xylose utilization in recombinant S. cerevisiae [10]; however, the degree to which transport controls the xylose uptake rate is dependent on both the substrate concentration in the medium and the downstream pathway. Prolonged chemostat cultivation at low xylose concentration selected for increased transport affinity [26], whereas transport had a negligible effect on xylose utilization at $>10 \mathrm{~g} / \mathrm{L}$ of xylose for most strains [8-10]. For this reason, many studies have failed to observe a physiological effect from overexpressing native or heterologous transporters [6,27]. Recently however, expression of xylose transporters from C. intermedia, P. stipitis and A. thaliana was reported to improve xylose utilization in recombinant $S$. cerevisiae $[9,11,12]$; however, different experimental designs and genetic constructions in these investigations limited a functional comparison of the

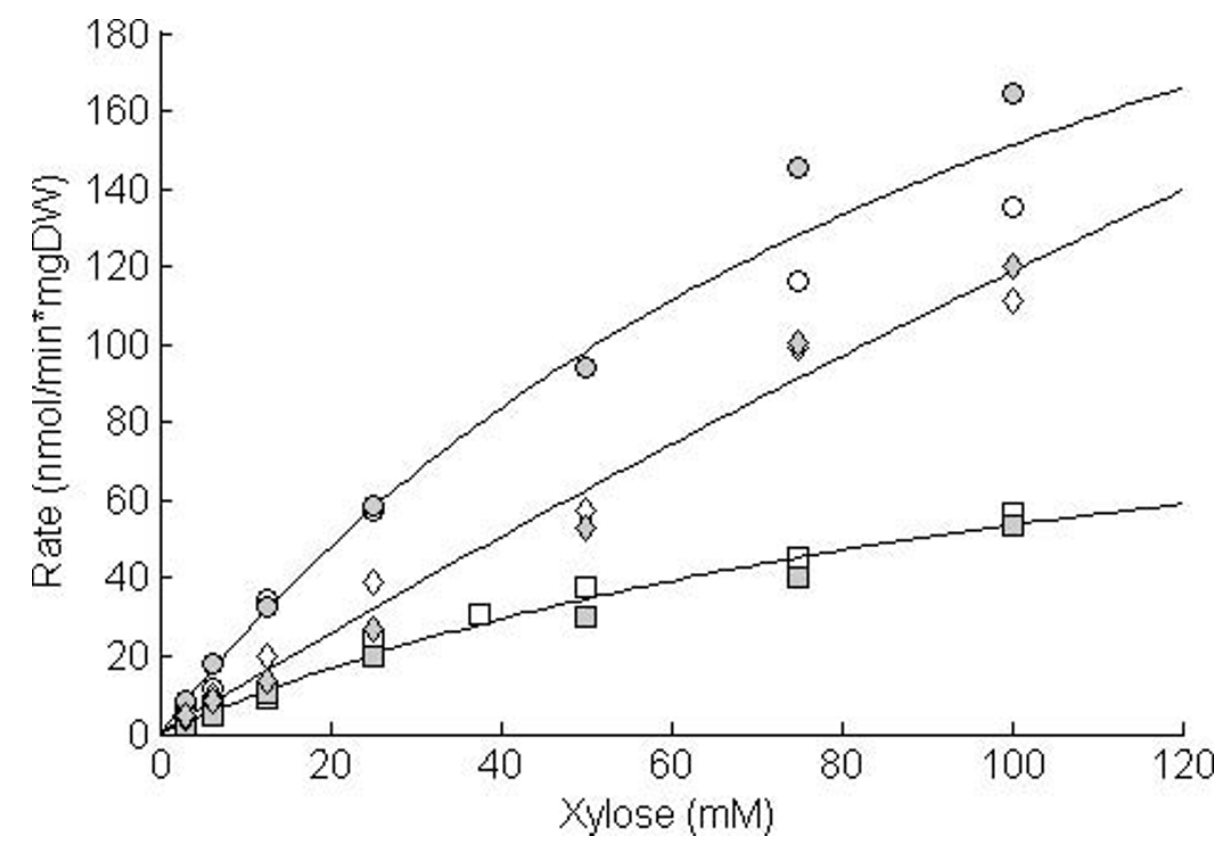

Figure 3 Xylose uptake rates of S. cerevisiae grown under different conditions. Lines represent the calculated fit according to MichaelisMenten kinetics. $20 \mathrm{~g} / \mathrm{L}$ Glucose, square; $0.5 \mathrm{~g} / \mathrm{L}$ glucose, circle; $60 \mathrm{~g} / \mathrm{L}$ xylose, diamond. Biological replicates are designated by empty and filled version of the same symbol. 
results. For instance, expression of the Sut1 [11] and At5g59250 transporters [12] showed enhanced utilization rate of glucose and xylose in batch culture, whereas expression of the Gxf1 transporter [9] improved xylose utilization only. For the Gxf1 transporter, improvement of xylose utilization was also restricted to substrate concentrations > $10 \mathrm{~g} / \mathrm{L}$ [9], whereas the Sut1 and At5g59250 transporters increased xylose uptake at substrate concentrations $>50 \mathrm{~g} / \mathrm{L}[11,12]$.

In the current study, expression of the Gxf1, Sut1 and At5g59250 transporters was compared in isogenic strains under identical conditions. Although expression of both the Gxf1 and the Sut1 transporter increased the xylose transport performance, the effect of the Gxf1 transporter was significantly more pronounced (Figure 1 . Table 2). In aerobic xylose cultivation at high and intermediate xylose concentrations (60 g/L and $15 \mathrm{~g} / \mathrm{L}$ xylose, respectively) none of the heterologous transporters increased the growth rate. The results confirm the role of extracellular xylose concentration in controlling the rate of xylose transport [8,9]. Furthermore, none of the transporters influenced the growth on glucose (results not shown), which was expected because glucose transport only controls the glycolytic rate at low glucose concentration [28]. However, during aerobic cultivation at $4 \mathrm{~g} / \mathrm{L}$ xylose, the measured transport kinetics were mirrored, in the sense that expression of the Gxf1 transporter led to the largest increase in growth rate, followed by the Sut1 transporter (Figure 2). Expression of the At5g59250 transporter did not increase xylose affinity and consequently the xylose growth rate was not affected (Figure 1, Figure 2).

The overall xylose transport performance of native $S$. cerevisiae was dependent on carbon source and concentration, and was highest under non-repressive conditions (xylose or low glucose) (Figure 3). Expression of native transporters clearly increased at low glucose concentrations relative to the constitutive expression of the heterologous transporters (Figure 1, Figure 3). The overall xylose transport in S. cerevisiae has been modeled in silico as a function of the extracellular glucose concentration [29]. In agreement with the current study, it was found that xylose transport was highest when the glucose concentration was $\sim 0.2-1 \mathrm{~g} / \mathrm{L}$. The influence of glucose on the regulation of xylose transport has similarly been demonstrated in simultaneous saccharification and fermentation, in which a low but steady glucose concentration is maintained during xylose utilization [30]. The industrial potential of expressing heterologous xylose transporters in S. cerevisiae is thus highly dependent on the concentration of both xylose and glucose in the process. For example, during batch operation the concentration of glucose and xylose are high and overexpression of repressed high-affinity native transporters may increase utilization of xylose. On the other hand, during fed-batch or continuous cultivation, the concentrations of glucose and xylose are low, which indicates that native high-affinity transporters are induced and expression of a heterologous transporter with superior xylose kinetics is needed to improve uptake.

Among the transporters investigated in this work, the Gxf1 transporter clearly improved the xylose transport capacity and growth rate more than the Sut1 and At5g59250 transporters (Figure 1, Table 2, Figure 2). An explanation for these results may be found in the way these transporters were originally isolated. The At5g59250 transporter was identified solely by sequence homology to known transporter genes and thus lacked a functional screening step [12]. On the other hand, the Sut1 transporter was identified by expression of a $P$. stipitis cDNA library in a $S$. cerevisiae $H X T$ null strain and subsequent reconstitution of growth on glucose [31]. By contrast, the Gxf1 transporter was isolated by comparing xylose growth in native xylose-utilizing yeast, including the aforementioned $P$. stipitis [32]. The highest xylose uptake was seen in the yeast $C$. intermedia, and subsequent screening of a C. intermedia cDNA library in an S. cerevisiae HXT null strain identified the GXF1 transporter gene [23]. Thus, the superior performance of the Gxf1 transporter demonstrated in the current study can probably be attributed to the original isolation process, which not only selected for functional expression in S. cerevisiae but also the highest transport capacity.

\section{Conclusions}

In the current study, the transporters Gxf1, Sut1 and At5g59250 were compared in isogenic S. cerevisiae strains under identical conditions. A direct relationship was seen between transport kinetics of the individual transporters and xylose utilization under transport limiting growth conditions. The relative xylose transport performance of native $S$. cerevisiae transporters was also found to be significantly increased during cultivation at low glucose concentrations, which points towards the importance of designing an appropriate cofermentation strategy for industrial ethanol production from lignocellulose biomass.

\section{Acknowledgements}

This work was supported by the Swedish Energy Agency

(Energimyndigheten). Camilla Freitag and Marit Emilsson are acknowledged for outstanding technical assistance.

\section{Authors' contributions}

DR participated in the design of the study, performed the experimenta work and wrote the manuscript. $\mathrm{BHH}$ participated in the design of the study and commented on the manuscript. PR participated in the design of the study and commented on the manuscript. All the authors have read and approved the final manuscript. 


\section{Competing interests}

The authors declare that they have no competing interests.

Received: 15 January 2010 Accepted: 17 March 2010

Published: 17 March 2010

\section{References}

1. Galbe M, Sassner P, Wingren A, Zacchi G: Process engineering economics of bioethanol production. Adv Biochem Engin/Biotechnol 2007, 108:303-327.

2. Hahn-Hägerdal B, Galbe M, Gorwa-Grauslund MF, Liden G, Zacchi G: Bioethanol-the fuel of tomorrow from the residues of today. Trends Biotechnol 2006, 24:549-556.

3. Hahn-Hägerdal B, Karhumaa K, Fonseca C, Spencer-Martins I, GorwaGrauslund MF: Towards industrial pentose-fermenting yeast strains. App/ Microbiol Biotechnol 2007, 74:937-953.

4. van Maris AJ, Winkler AA, Kuyper M, de Laat WT, van Dijken JP, Pronk JT: Development of efficient xylose fermentation in Saccharomyces cerevisiae : xylose isomerase as a key component. Adv Biochem Engin/ Biotechnol 2007, 108:179-204.

5. Jeffries TW: Engineering yeasts for xylose metabolism. Curr Opin Biotechnol 2006, 17:320-326.

6. Saloheimo A, Rauta J, Stasyk OV, Sibirny AA, Penttila M, Ruohonen L: Xylose transport studies with xylose-utilizing Saccharomyces cerevisiae strains expressing heterologous and homologous permeases. Appl Microbiol Biotechnol 2007, 74:1041-1052.

7. Lee WJ, Kim MD, Ryu YW, Bisson LF, Seo JH: Kinetic studies on glucose and xylose transport in Saccharomyces cerevisiae. Appl Microbiol Biotechnol 2002, 60:186-191.

8. Gárdónyi M, Jeppsson M, Liden G, Gorwa-Grauslund MF, Hahn-Hägerdal B: Control of xylose consumption by xylose transport in recombinant Saccharomyces cerevisiae. Biotechnol Bioeng 2003, 82:818-824.

9. Runquist D, Fonseca C, Radstrom P, Spencer-Martins I, Hahn-Hägerdal B: Expression of the Gxf1 transporter from Candida intermedia improves fermentation performance in recombinant xylose-utilizing Saccharomyces cerevisiae. Appl Microbiol Biotechnol 2009, 82:123-130.

10. Kötter P, Ciriacy M: Xylose fermentation by Saccharomyces cerevisiae. Appl Microbiol Biotechnol 1993, 38:776-783.

11. Katahira S, Ito M, Takema H, Fujita Y, Tanino T, Tanaka T, Fukuda H, Kondo A: Improvement of ethanol productivity during xylose and glucose co-fermentation by xylose-assimilating $S$. cerevisiae via expression of glucose transporter Sut1. Enzyme Microb Technol 2008, 43:115-119.

12. Hector RE, Qureshi N, Hughes SR, Cotta MA: Expression of a heterologous xylose transporter in a Saccharomyces cerevisiae strain engineered to utilize xylose improves aerobic xylose consumption. Appl Microbiol Biotechnol 2008, 80:675-684.

13. Verduyn C, Postma E, Scheffers WA, Van Dijken JP: Energetics of Saccharomyces cerevisiae in anaerobic glucose-limited chemostat cultures. J Gen Microbiol 1990, 136:405-412.

14. Hahn-Hägerdal B, Karhumaa K, Larsson CU, Gorwa-Grauslund M, Gorgens J, van Zyl WH: Role of cultivation media in the development of yeast strains for large scale industrial use. Microb Cell Fact 2005, 4:31.

15. Sambrook J, Fritsch EF, Maniatis T: Molecular Cloning: A Laboratory Manual Cold Spring Harbor: Cold Spring Harbor Laboratory Press, 21989.

16. Inoue $\mathrm{H}$, Nojima $\mathrm{H}$, Okayama $\mathrm{H}$ : High efficiency transformation of Escherichia coli with plasmids. Gene 1990, 96:23-28.

17. Gietz RD, Schiestl RH, Willems AR, Woods RA: Studies on the transformation of intact yeast cells by the LiAc/s-DNA/PEG procedure. Yeast 1995, 11:355-360

18. Grote A, Hiller K, Scheer M, Munch R, Nortemann B, Hempel DC, Jahn D: JCat: a novel tool to adapt codon usage of a target gene to its potential expression host. Nucleic Acids Res 2005, 33:W526-531.

19. Bettiga M, Bengtsson O, Hahn-Hägerdal B, Gorwa-Grauslund MF: Arabinose and xylose fermentation by recombinant Saccharomyces cerevisiae expressing a fungal pentose utilization pathway. Microb Cell Fact 2009, 8:40.

20. Bisson $L F$, Fraenkel DG: Involvement of kinases in glucose and fructose uptake by Saccharomyces cerevisiae. Proc Natl Acad Sci USA 1983, 80:1730-1734.

21. Eisenthal R, Danson MJ, Hough DW: Catalytic efficiency and kcat/KM: a useful comparator? Trends Biotechnol 2007, 25:247-249.
22. Koshland DE: The application and usefulness of the ratio $k(c a t) / K(M)$. Bioorg Chem 2002, 30:211-213.

23. Leandro MJ, Goncalves P, Spencer-Martins I: Two glucose/xylose transporter genes from the yeast Candida intermedia : first molecular characterization of a yeast xylose-H+ symporter. Biochem J 2006, 395:543-549.

24. Fell DA: Understanding the Control of Metabolism London: Portland Press 1997.

25. Sedlak M, Ho NW: Characterization of the effectiveness of hexose transporters for transporting xylose during glucose and xylose cofermentation by a recombinant Saccharomyces yeast. Yeast 2004, 21:671-684.

26. Kuyper M, Toirkens MJ, Diderich JA, Winkler AA, van Dijken JP, Pronk JT: Evolutionary engineering of mixed-sugar utilization by a xylosefermenting Saccharomyces cerevisiae strain. FEMS Yeast Res 2005, 5:925-934

27. Hamacher T, Becker J, Gárdónyi M, Hahn-Hägerdal B, Boles E: Characterization of the xylose-transporting properties of yeast hexose transporters and their influence on xylose utilization. Microbiology 2002, 148:2783-2788.

28. Elbing K, Larsson C, Bill RM, Albers E, Snoep JL, Boles E, Hohmann S, Gustafsson L: Role of hexose transport in control of glycolytic flux in Saccharomyces cerevisiae. Appl Environ Microbiol 2004, 70:5323-5330.

29. Bertilsson M, Andersson J, Liden G: Modeling simultaneous glucose and xylose uptake in Saccharomyces cerevisiae from kinetics and gene expression of sugar transporters. Bioprocess Biosystems Eng 2008, 31:369-377.

30. Olofsson K, Bertilsson M, Liden G: A short review on SSF - an interesting process option for ethanol production from lignocellulosic feedstocks. Biotechnol Biofuels 2008, 1:7.

31. Weierstall $T$, Hollenberg $C P$, Boles E: Cloning and characterization of three genes (SUT1-3) encoding glucose transporters of the yeast Pichia stipitis. Mol Microbiol 1999, 31:871-883.

32. Gárdónyi M, Osterberg M, Rodrigues C, Spencer-Martins I, Hahn-Hägerdal B: High capacity xylose transport in Candida intermedia PYCC 4715. FEMS Yeast Res 2003, 3:45-52.

33. Gietz RD, Sugino A: New yeast-Escherichia coli shuttle vectors constructed with in vitro mutagenized yeast genes lacking 6-base pair restriction sites. Gene 1988, 74:527-534.

34. Karhumaa K, Hahn-Hägerdal B, Gorwa-Grauslund MF: Investigation of limiting metabolic steps in the utilization of xylose by recombinant Saccharomyces cerevisiae using metabolic engineering. Yeast 2005, 22:359-368.

doi:10.1186/1754-6834-3-5

Cite this article as: Runquist et al:: Comparison of heterologous xylose transporters in recombinant Saccharomyces cerevisiae. Biotechnology for Biofuels 2010 3:5.

\section{Submit your next manuscript to BioMed Central and take full advantage of:}

- Convenient online submission

- Thorough peer review

- No space constraints or color figure charges

- Immediate publication on acceptance

- Inclusion in PubMed, CAS, Scopus and Google Scholar

- Research which is freely available for redistribution
Biomed Central 